\title{
Design, Synthesis, and Characterization of Novel Thiol-Derivatized Ibuprofen Monolayer Protected Gold Clusters
}

\author{
Kuan-Han Lee, ${ }^{1,2}$ Yu-Sheng Lin, ${ }^{3}$ and Po-Jui Huang ${ }^{3}$ \\ ${ }^{1}$ Department of Pharmacy, Chia Nan University of Pharmacy and Science, Tainan 71710, Taiwan \\ ${ }^{2}$ Drug Discovery and Development Center, Chia Nan University of Pharmacy and Science, Tainan 71710, Taiwan \\ ${ }^{3}$ Department of Medicinal and Applied Chemistry, Kaohsiung Medical University, Kaohsiung 80708, Taiwan
}

Correspondence should be addressed to Po-Jui Huang; brhuang@kmu.edu.tw

Received 24 December 2012; Revised 31 January 2013; Accepted 14 February 2013

Academic Editor: Zhenhui Kang

Copyright (C) 2013 Kuan-Han Lee et al. This is an open access article distributed under the Creative Commons Attribution License, which permits unrestricted use, distribution, and reproduction in any medium, provided the original work is properly cited.

A series of new thiol-derivatized ibuprofen monolayer protected gold clusters have been prepared by amidation of ibuprofen with alkyl alcohol or aminophenol affording the carboxamides, $\mathrm{N}$-hydroxyalkyl amide $\mathbf{2}$, and $\mathrm{N}$-hydroxyphenyl amide $\mathbf{6}$, which were then tosylated with $p$-toluenesulfonyl chloride at hydroxyl group to give $\mathbf{3}$ and $\mathbf{7}$. Reactions of $\mathbf{3}$ and $\mathbf{7}$ with $\mathrm{NaSH}$ afforded the mercapto derivatives 4 and $\mathbf{8}$. Conducting Brust's reaction with a $3: 1$ mole ratio of thiolate ibuprofen/ $\mathrm{AuCl}_{4}{ }^{-}$yielded polydisperse thiol-derivatized ibuprofen-MPCs $\mathbf{5}$ and $\mathbf{9}$. All compounds have been identified by NMR, MS, UV, and IR spectroscopies. Compounds $\mathbf{4}$ and $\mathbf{8}$ and the MPCs $\mathbf{5}$ and $\mathbf{9}$ have been investigated by using the method of ${ }^{1} \mathrm{H}$ NMR spectroscopy. The broadening of the signals from 0.8 to 2.0 ppm in ${ }^{1} \mathrm{H}$ NMR spectrum of MPCs $\mathbf{5}$ and $\mathbf{9}$ confirmed the success of the conjugation of thiol-containing derivatives with nanogold cluster.

\section{Introduction}

Gold nanoparticles have been the subject of increasing attention for the past decade, particularly since Brust et al. introduced a simple method for the preparation of monodisperse gold nanoparticles stabilized with various thiol-containing ligands [1]. Gold thiolates have been the principal compounds used in chrysotherapy, involving the treatment of rheumatoid arthritis (RA) with gold-based drugs [2]. When delivered as gold nanoconjugates, these drugs may have a better efficiency with reduced systemic toxicity. Moreover, the nanoscale size of these bioconjugates is helpful to prevent uptake by mononuclear phagocytic cells and allow for their penetration through the smallest capillary pores within the human vascular [3]. The lack of toxicity has made gold a logical and practical choice to be used as a vehicle for drug targeting [4, 5]. Recently, there has been an increasing use of gold nanoparticles (GNPs) in various biomedical applications $[6,7]$. As the most stable metallic particles, GNPs are highly attractive because of their distinct surface plasmon resonance (SP) bands which provide easy characterization, incorporating functional ligands to create monolayer protected gold clusters (MPCs) and their biocompatibility [8].

To our knowledge, this is the first attempt in attaching ibuprofen to GNPs to form MPCs. Only a few reports described the loading of ibuprofen into the porous magnetic hollow silica nanosphere (MHSNs) to study their drug loading and control releasing capabilities [9]. Here, we report the first example of the preparation of ibuprofen-MPCs. GNPs were thermally reshaped to homogenize the size of particles [10-12]. By using the functional group modification method, the ibuprofen was attached to GNPs, followed by the Brust's reaction to afford ibuprofen-MPCs [13]. We believe that it is an important result in the synthesis of ibuprofen-MPCs as a new category of nano-NSAIDs (Scheme 1).

\section{Materials and Methods}

2.1. Materials. All manipulations involving air-sensitive materials were carried out by using standard Schlenk techniques under an atmosphere of $\mathrm{N}_{2}$. Reagents were purchased 


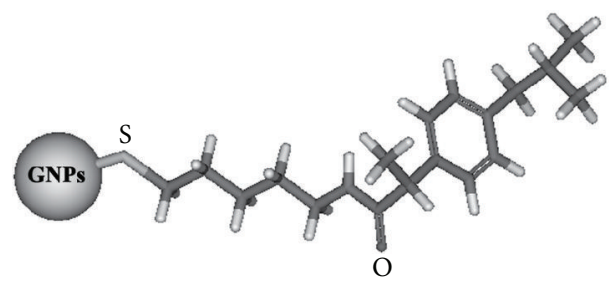

(a)

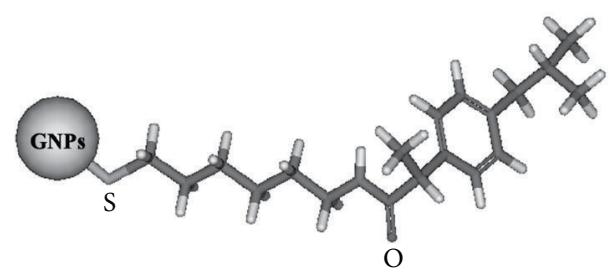

(b)

FIGURE 1: $3 \mathrm{D}$ structure for $\mathbf{5} \mathbf{a}$ and $\mathbf{5 b}$ after energy-minimization, with thiol $(\mathrm{S})$ and amide $(\mathrm{C}=\mathrm{O})$ atoms indicated.

from commercial sources and used without further purification. Solvents were purified and dried by a standard procedure before use. All other chemicals and reagents used were of analytical grade.

\subsection{Methods}

2.2.1. Characterization Methods. Melting points were determined on a Yanaco micromelting-point apparatus and are uncorrected. Nuclear magnetic resonance (NMR) $\left({ }^{1} \mathrm{H}\right.$ and

$\left.{ }^{13} \mathrm{C}\right)$ spectra were recorded on a Varian UNITY plus-400 FT spectrometer. Chemical shifts were expressed in parts per million (ppm) with tetramethylsilane (TMS) as an internal standard. The UV-VIS spectra were obtained with a Hitachi U-4000 spectroscope. IR spectra were obtained on a BioRad FT-IR spectrophotometer with samples prepared as $\mathrm{KBr}$ pellets. The ESI-MS was recorded on Bruker APEX II FT mass spectrometer. Morphologies of all samples were investigated by Philip CM-200 TWIN Transmission Electron Microscope (TEM). A drop of dilute solution of sample (1 $\mathrm{mg}$ sample in $1 \mathrm{~mL} n$-hexane) was dropped into a 200-mesh carbon-coated copper grid sample holder. Excess solvent was swept away by touching the edge of the grids with a small piece of filter paper. The grids were dried in vacuum and directly observed by TEM.

2.2.2. Synthetic Approaches to Thiol-Derivatized Monolayer Protected Gold Clusters (MPCs) 5 and 9. A majority of the alkyl chains are in all-trans conformation. The amide side chains at varying positions have a dramatic influence in the hydrogen-bonding efficiency. The radial dependence provides a means of controlling the assembly of the protecting monolayer. In this study, we chose pentyl and hexyl moieties as carbon side chains $(9.32 \AA$ and $10.45 \AA$ measured from thiol to amide group, resp.) in compounds $\mathbf{5 a}$ and $\mathbf{5 b}$ to modulate the hydrogen-bonding radial structure (Figure 1). Furthermore, the arene functionality was also incorporated

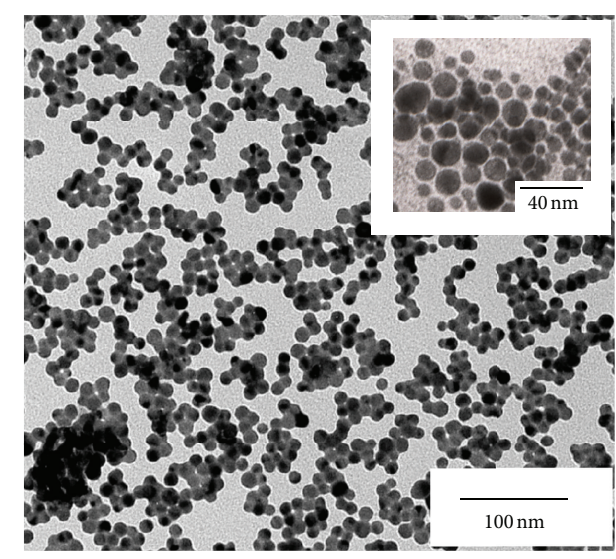

(a)

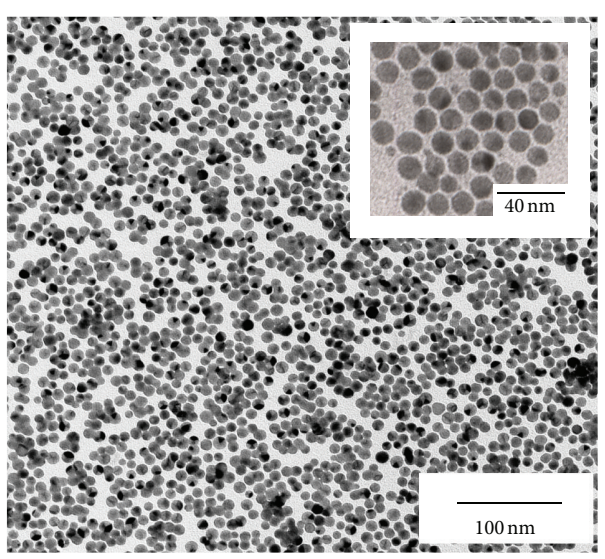

(b)

FIGURE 2: TEM micrograph of thiol-derivatized ibuprofen-MPCs samples: (a) nonuniform $9 \pm 1 \mathrm{~nm}$ sized particles prepared by standard two-phase protocol and (b) homogenized $13 \pm 1 \mathrm{~nm}$ gold particle samples prepared by heating treatment at $190^{\circ} \mathrm{C}$. Inset photograph shows the selected area of representative image of MPCs samples.

into the side chain in compound 9 to probe the influence of the nature of the side chain on the MPCs. Preparation of the target compounds $\mathbf{5}$ and $\mathbf{9}$ is illustrated in Schemes 2 and 3. Amidation of ibuprofen with alkyl alcohol or aminophenol provided the carboxamides, $\mathrm{N}$-hydroxyalkyl amide 2 , and $N$-hydroxyphenyl amide $\mathbf{6}$, which were then tosylated with $p$-toluenesulfonyl chloride at hydroxyl group to afford $\mathbf{3}$ and 7 . Reactions of $\mathbf{3}$ and $\mathbf{7}$ with $\mathrm{NaSH}$ afforded the mercapto derivatives $\mathbf{4}$ and $\mathbf{8}$. Conducting Brust's reaction with a $3: 1$ mole ratio of thiolate ibuprofen $/ \mathrm{AuCl}_{4}{ }^{-}$yielded a polydisperse thiol-derivatized ibuprofen-MPCs $\mathbf{5}$ and $\mathbf{9}$ in $90 \%$ yield. The experimental details and characterization of compounds 2-9 $\left({ }^{1} \mathrm{HNMR},{ }^{13} \mathrm{CNMR}\right.$, and HRMS) are provided in the Supplementary Materials available online at http://dx.doi.org/10.1155/2013/931815.

\section{Results and Discussion}

3.1. Characterizations of the Morphology and Structural Properties. The heating-evolved nanoparticles were characterized 


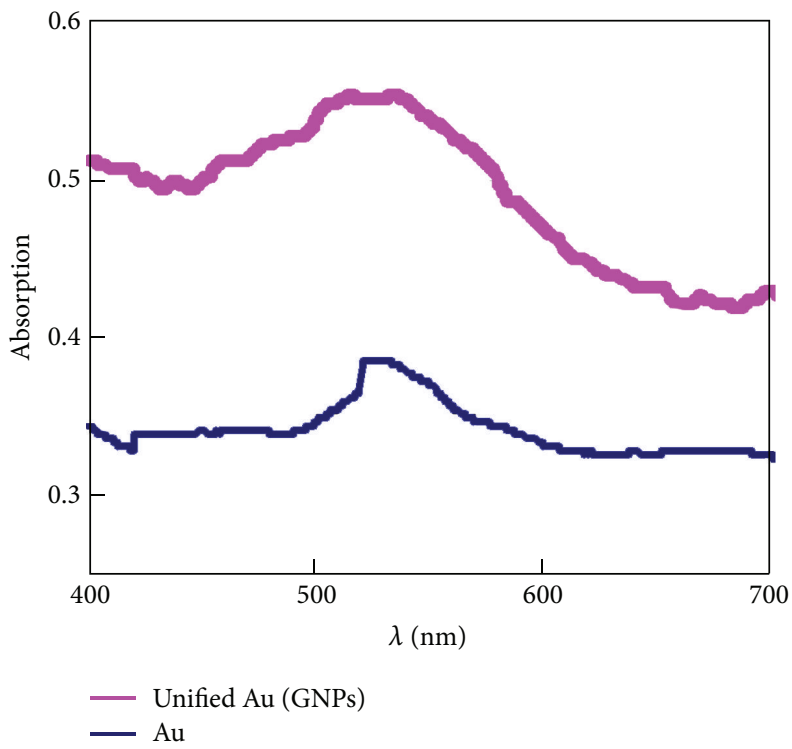

(a)
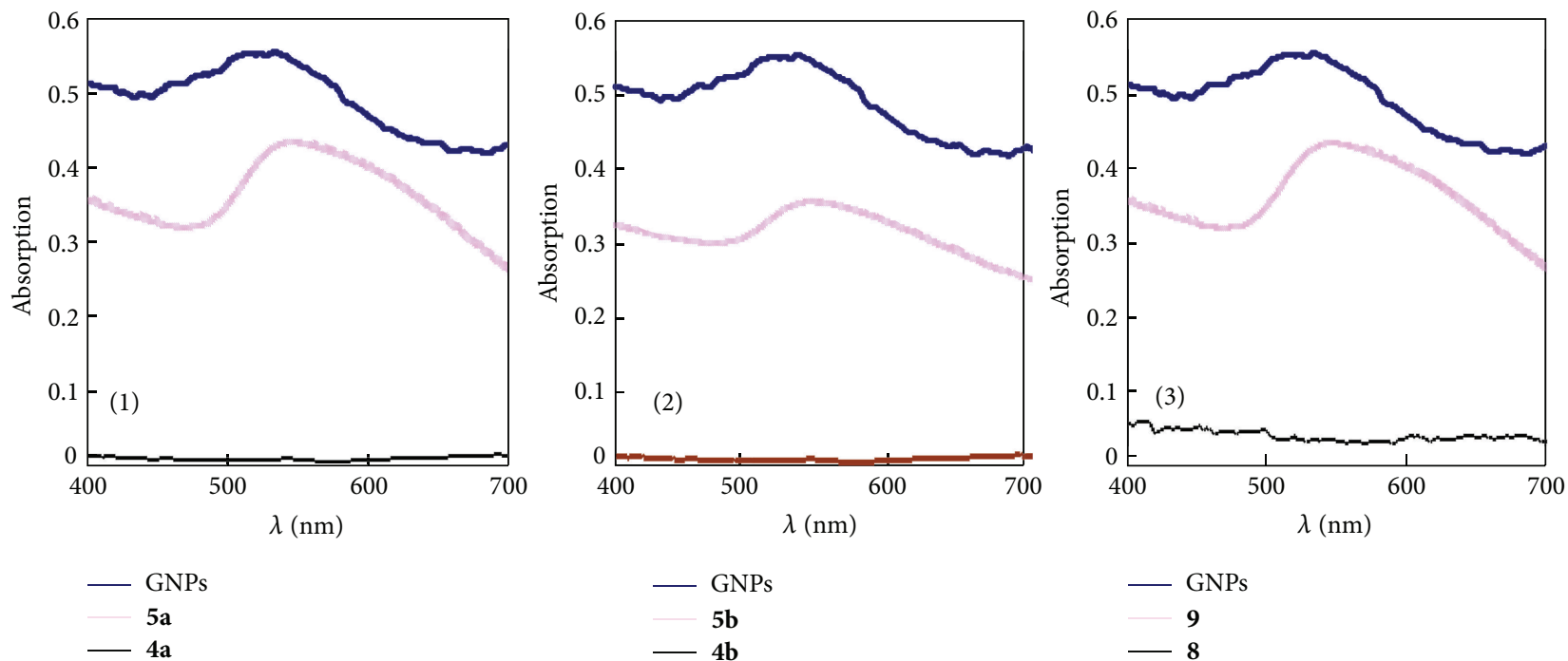

(b)

Figure 3: UV-VIS spectra of (a) Au and unified gold nanoparticles (GNPs). (b) (1) GNPs, 4a, and MPC 5a. (2) GNPs, 4b, and MPC 5b. (3) GNPs, 8, and MPC 9.

by a number of techniques: TEM was used for the measurement of particle size and shape; UV-VIS was used for size evolution and monitoring the plasmon absorption of the produced compounds; IR and NMR were used for the elucidation of thiolate shell structures.

3.1.1. TEM Imaging. Nanoparticles synthesized according to the standard two-phase protocol and characterized by TEM were reported in the recent literatures $[1,14,15]$. The TEM data showed an average core size of $9 \pm 1 \mathrm{~nm}$ with $\sim 80 \%$ populations. As shown in Figure 2(a), the particle sizes appeared nonuniform. In contrast to the morphological features, several striking observations are evident for the nanoparticles evolving from the heat treatment, as shown in
Figure 2(b) for a representative TEM image. The TEM image displays an increased core size with the narrow distribution in which more than $90 \%$ populations are at the $13 \pm 1 \mathrm{~nm}$ range. The distribution of such evolved particles is much narrower than that of the $9 \pm 1 \mathrm{~nm}$ particles without heat treatment.

3.1.2. UV-VIS Measurement. UV-VIS absorption spectroscopy was used to monitor the plasmon absorption of the produced MPCs. Both intensity and energy of the surface plasmon resonance (SP) bands of nanoparticles are known to be strongly dependent on size [16]. We thus carried out UV-VIS measurement to access the size evolution of the nanoparticles in solution. The spectrum for the $9 \pm 1 \mathrm{~nm}$ sized Au particles displays an identifiable but weak SP band 


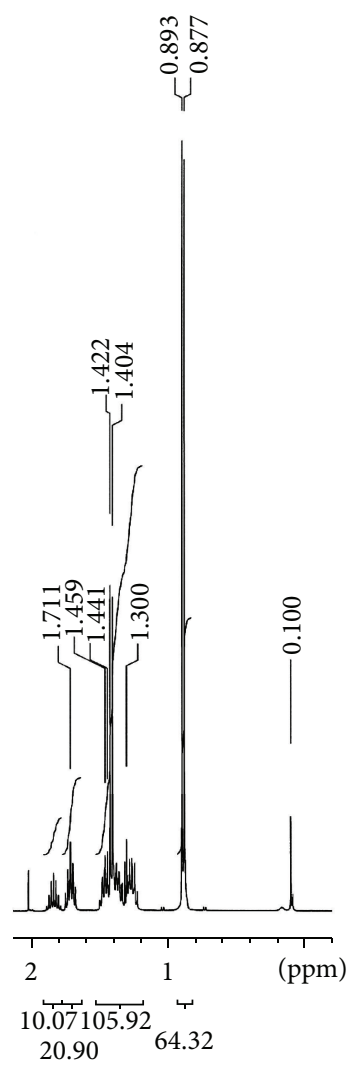

(a)

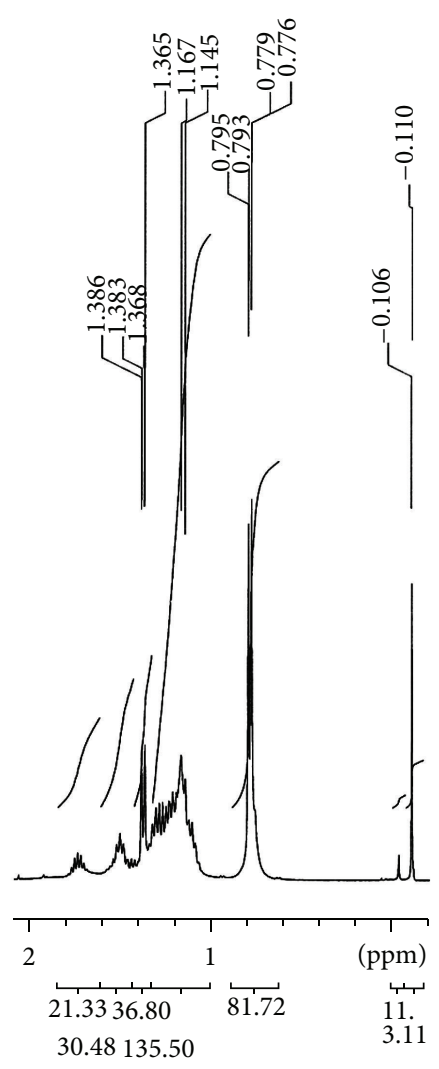

(b)
FIGURE 4: Selected area of ${ }^{1} \mathrm{H}$ NMR spectra of (a) thiol-derivatized ibuprofen samples $\mathbf{4} \mathbf{b}$ and (b) thiol-derivatized ibuprofen-MPCs sample $5 \mathbf{b}$.

enveloped around $515 \mathrm{~nm}$. In contrast, the spectra of the unified Au (GNPs) after the heat treatment at $190^{\circ} \mathrm{C}$ exhibited an increased intensity of the SP band at $520 \mathrm{~nm}$. The increased intensity of the SP band is indicated by an increase in core size (Figure 3(a)) [17].

Figure 3(b) shows the absorption spectrum of GNPs, thiol-derivatized compounds (4 and 8), and MPCs (5 and 9) in $n$-hexane. The UV absorption of the GNPs and MPCs decays roughly exponentially into the visible, with an SP band located at $\sim 520 \mathrm{~nm}$. It is a typical characteristic of particulate gold solution ascribed to a collective oscillation of the conductive electrons in response to optical excitation. However, thiol-derivatized compounds only show a very weak absorption under the same condition.

3.1.3. NMR Spectroscopic Analysis. NMR spectroscopy is particularly informative about the structure and content of gold nanoparticles $[15,18,19]$. NMR resonances of gold nanoparticles are characteristically broadened relative to those of free alkanethiols; factors include broadening of the spin-spin relaxation time ( $T 2$, also known as transverse relaxation or relaxation in the $x-y$ plane), a distribution in chemical shifts due to differences in GNPs-SR binding sites, and a gradient in monolayer packing density from nearcore to chain terminus with associated dipolar broadening. Figure 4(b) illustrates the broadening of ${ }^{1} \mathrm{H}$ NMR resonances of thiol-derivatized ibuprofen-MPCs with increased core size, which for the methyl resonance $(\sim 0.8 \mathrm{ppm})$ is thought to reflect primarily T2 broadening.

The solution NMR technique was used to confirm an important question, which was whether $\mathbf{5}$ and $\mathbf{9}$ functionalized the organic shell or not. Figure 4 shows the ${ }^{1} \mathrm{H}$ NMR spectra for the $\mathbf{4 b}$ and ibuprofen-functionalized MPCs $\mathbf{5 b}$. The high field $(0.8-2.3 \mathrm{ppm})$ peaks established the resonances as contributed from the $-\mathrm{CH}_{2}$ and $-\mathrm{CH}_{3}$ groups. It was interesting to note that the ibuprofen alkanethiol 4 (Figure 4(a)) gave sharper NMR signals at room temperature in comparison with the ibuprofen-functionalized MPCs $\mathbf{5 b}$ (Figure $4(\mathrm{~b})$ ). The conformation of the functionalization comes from the low field peaks at 6.0-8.0 ppm, which established the resonances of the ibuprofenyl amino alkanol moieties.

3.1.4. IR Spectroscopic Analysis. IR spectroscopy has been used frequently for the characterization of the as-synthesized thiolate-encapsulated gold nanoparticles and any kind of interaction between thiolate-derivatized samples and GNPs $[20,21]$. The infrared spectra of MPCs were collected over the range from 400 to $4000 \mathrm{~cm}^{-1}$. The IR spectra obtained for the GNPs, thiolate-derivatized ibuprofen $\mathbf{8}$, and MPC 9 are shown in Figure 5. Comparing the overall similarity of IR spectra in both high- and low-energy regions, it suggests the absence of major structural decomposition or transformation. The IR spectra of $\mathbf{8}$ and $\mathbf{9}$ show absorptions around 3330,3460 , and $1645 \mathrm{~cm}^{-1}$ which are attributed to the stretching vibrations of $\mathrm{N}-\mathrm{H}$ of the amide and $\mathrm{C}=\mathrm{O}$ groups, respectively. The alkyl side chains $\mathrm{C} 4$ or greater, as indicated by the asymmetric $\mathrm{CH}_{2}$ stretches, lie around $2920 \mathrm{~cm}^{-1}$ and symmetric stretching bands at $2850 \mathrm{~cm}^{-1}$. The finger regions and all the other bands of $\mathbf{8}$ were observed in the spectra of MPC 9. The SH stretching vibration of free alkanethiols is found at around $2400 \mathrm{~cm}^{-1}$. However, none of the SH stretching vibrations are found in the MPCs spectra. The data suggest that $\mathrm{SH}$ bond of the alkanethiol is broken upon binding to the GNPs.

\section{Conclusions}

A series of gold MPCs containing ibuprofen moiety was studied. Thiol-derivatized ibuprofen-MPCs were synthesized by Brust's method and characterized by a variety of analytical techniques. In this study, we believe that our findings provide a new pathway for the production of ibuprofen-MPCs with well-defined size and shape from preformed nanoparticles by a relatively simple heating procedure. The versatile and reproducible procedure may be extended to many other similar composite nanoparticle systems. The resulting thiolderivatized ibuprofen-MPCs could be a promising candidate for the treatment of rheumatoid arthritis. 


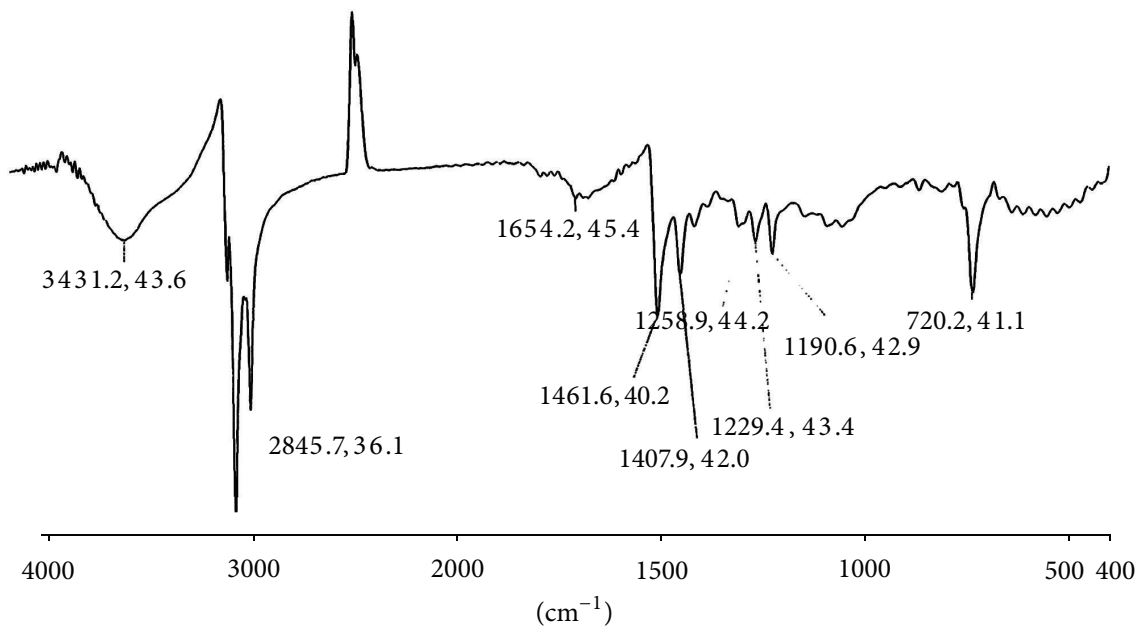

(a)

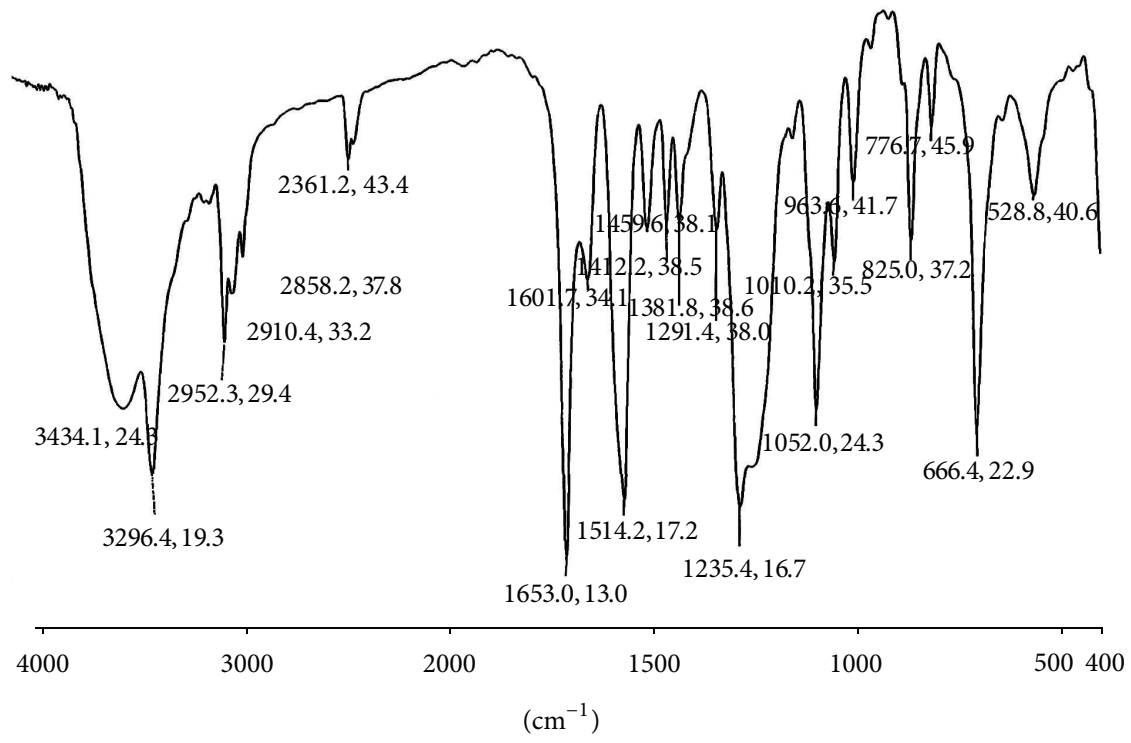

(b)

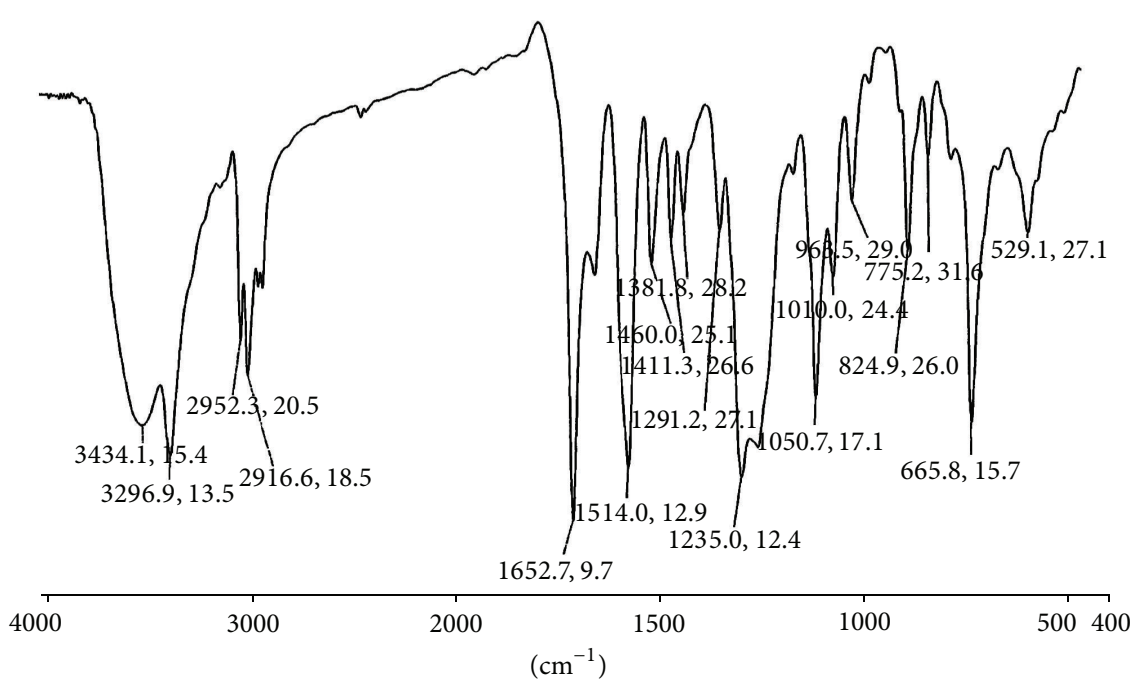

(c)

FIGURE 5: IR spectroscopic analysis of (a) GNPs. (b) Thiol-derivatized ibuprofen 8. (c) Thiol-derivatized ibuprofen-MPC 9. 

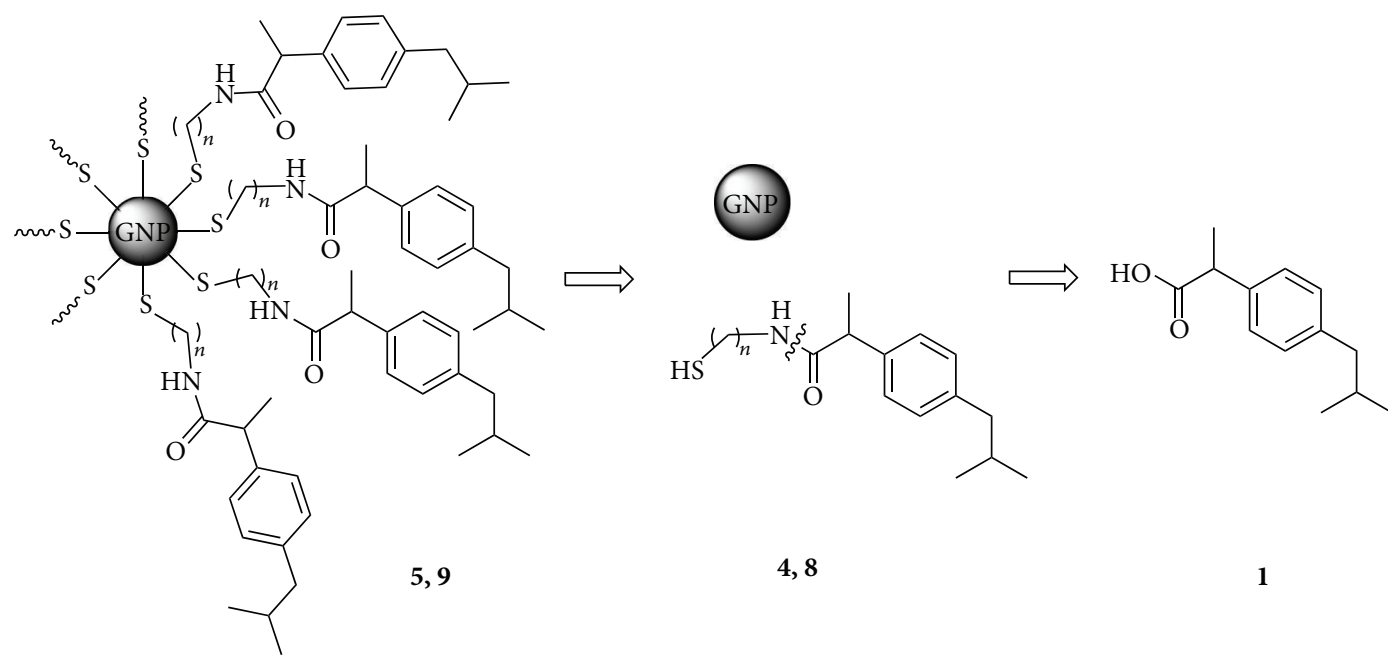

Scheme 1: Schematic representation of retrosynthetic analysis for thiol-derivatized ibuprofen-MPCs.

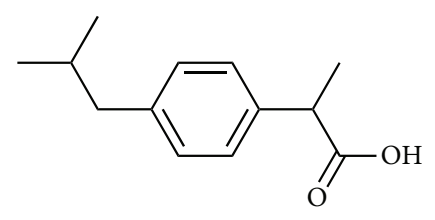

1

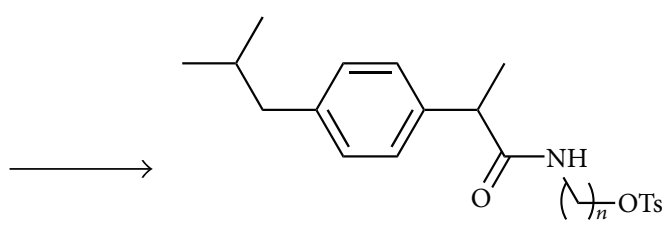

3

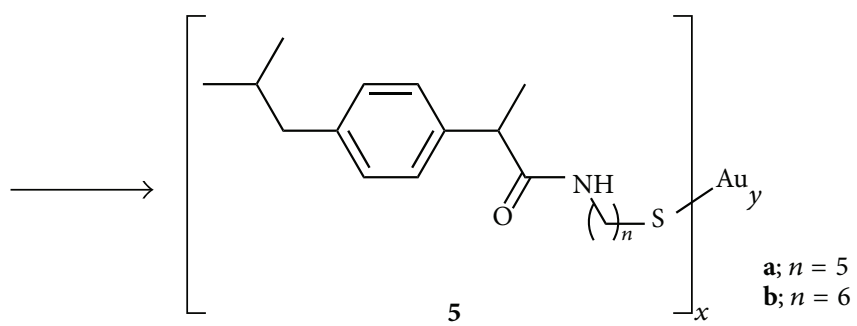

Scheme 2: Synthetic pathway for the preparation of title compounds 5
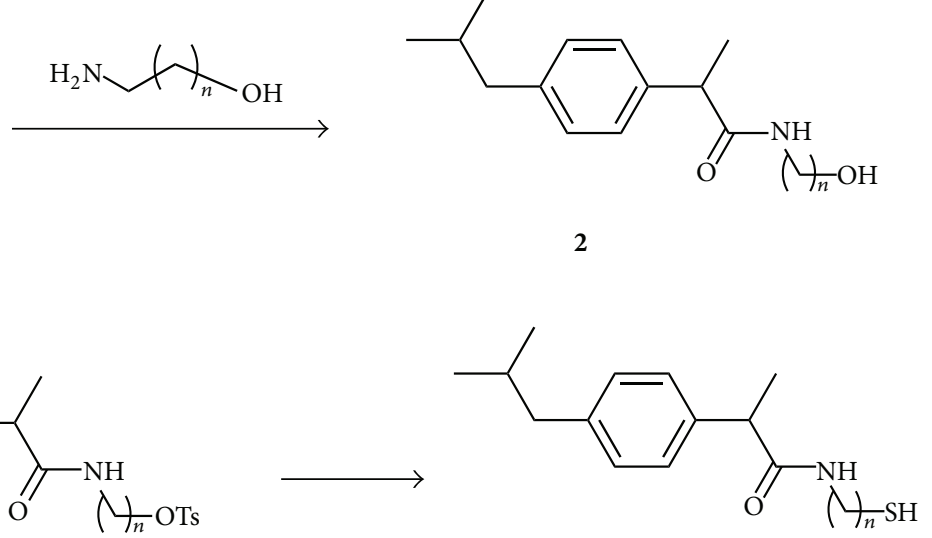

4 


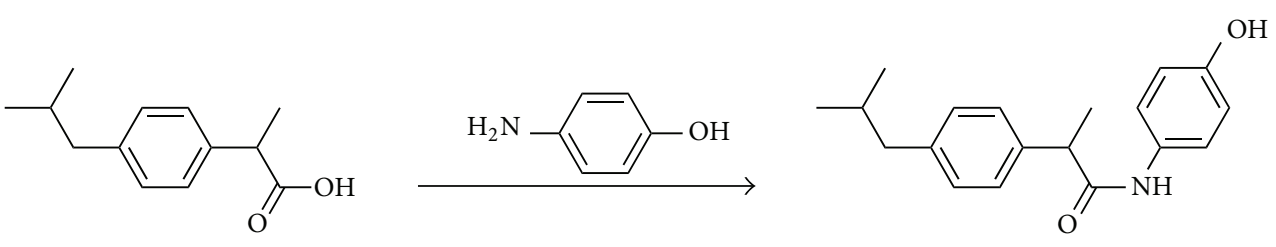

1

6

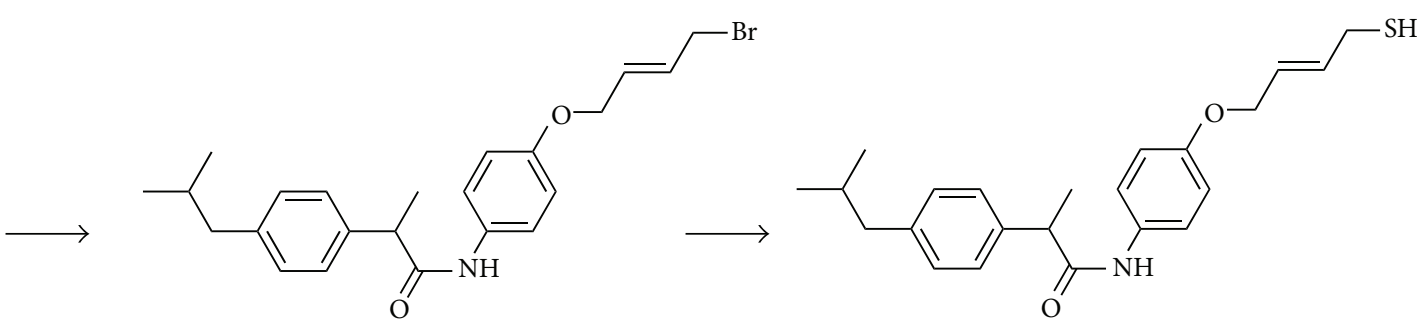

7

8

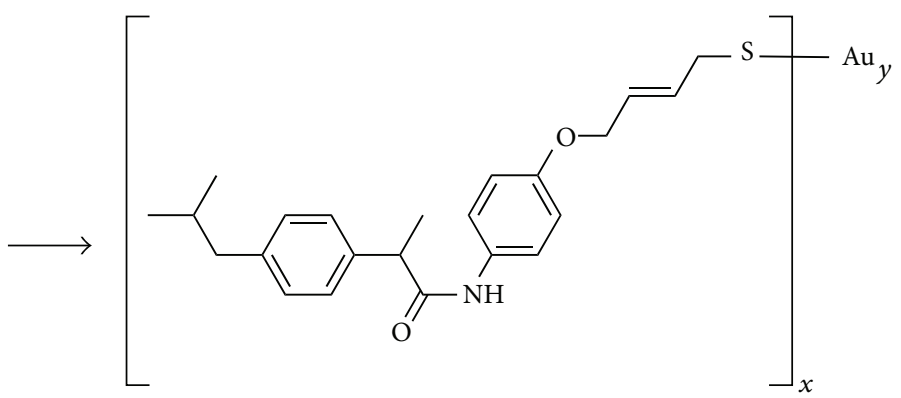

9

SCHeme 3: Synthetic pathway for the preparation of title compounds 9.

\section{Acknowledgments}

The authors are grateful to the National Science Council, Kaohsiung Medical University, and Chia Nan University of Pharmacy \& Science for financial support. The authors also wish to thank Professor Teng-Yuan Dong, Department of Chemistry, National Sun-Yen-Sun University, Taiwan, for his helpful discussion and constructive input to this paper.

\section{References}

[1] M. Brust, M. Walker, D. Bethell, D. J. Schiffrin, and R. Whyman, "Synthesis of thiol-derivatised gold nanoparticles in a twophase liquid-liquid system," Journal of the Chemical Society, Chemical Communications, no. 7, pp. 801-802, 1994.

[2] D. Gordon, G. H. Beastall, J. A. Thomson, and R. D. Sturrock, "Prolonged hypogonadism in male patients with rheumatoid arthritis during flares in disease activity," British Journal of Rheumatology, vol. 27, no. 6, pp. 440-444, 1988.

[3] A. S. Zahr, M. De Villiers, and M. V. Pishko, "Encapsulation of drug nanoparticles in self-assembled macromolecular nanoshells," Langmuir, vol. 21, no. 1, pp. 403-410, 2005.
[4] M. Thomas and A. M. Klibanov, "Conjugation to gold nanoparticles enhances polyethylenimine's transfer of plasmid dna into mammalian cells," Proceedings of the National Academy of Sciences of the United States of America, vol. 100, no. 16, pp. 91389143, 2003 .

[5] C. M. Goodman, C. D. McCusker, T. Yilmaz, and V. M. Rotello, "Toxicity of gold nanoparticles functionalized with cationic and anionic side chains," Bioconjugate Chemistry, vol. 15, no. 4, pp. 897-900, 2004.

[6] X. Huang, I. H. El-Sayed, W. Qian, and M. A. El-Sayed, "Cancer cell imaging and photothermal therapy in the nearinfrared region by using gold nanorods," Journal of the American Chemical Society, vol. 128, no. 6, pp. 2115-2120, 2006.

[7] L. R. Hirsch, R. J. Stafford, J. A. Bankson et al., "Nanoshellmediated near-infrared thermal therapy of tumors under magnetic resonance guidance," Proceedings of the National Academy of Sciences of the United States of America, vol. 100, no. 23, pp. 13549-13554, 2003.

[8] Y. Y. Huang, H. Y. Hsu, and C. J. C. Huang, "A protein detection technique by using surface plasmon resonance (SPR) with rolling circle amplification (RCA) and nanogold-modified tags," Biosensors and Bioelectronics, vol. 22, no. 6, pp. 980-985, 2007. 
[9] J. Zhou, W. Wu, D. Caruntu et al., "Synthesis of porous magnetic hollow silica nanospheres for nanomedicine application," Journal of Physical Chemistry C, vol. 111, no. 47, pp. 17473-17477, 2007.

[10] M. M. Maye, Y. Lou, and C. J. Zhong, "Core-shell gold nanoparticle assembly as novel electrocatalyst of CO oxidation," Langmuir, vol. 16, no. 19, pp. 7520-7523, 2000.

[11] C. J. Zhong, W. X. Zhang, F. L. Leibowitz, and H. H. Eichelberger, "Size and shape evolution of core-shell nanocrystals," Chemical Communications, no. 13, pp. 1211-1212, 1999.

[12] C. J. Zhong, W. X. Zheng, and F. L. Leibowitz, "Electrode nanomaterials self-assembled from thiolate-encapsulated gold nanocrystals," Electrochemistry Communications, vol. 1, no. 2, pp. 72-77, 1999.

[13] X. Liu, J. G. Worden, Q. Huo, and J. P. Brennan, "Kinetic study of gold nanoparticle growth in solution by Brust-Schiffrin reaction," Journal of Nanoscience and Nanotechnology, vol. 6, no. 4, pp. 1054-1059, 2006.

[14] M. Brust, J. Fink, D. Bethell, D. J. Schiffrin, and C. Kiely, "Synthesis and reactions of functionalized gold nanoparticles," Journal of the Chemical Society-Chemical Communications, no. 16, pp. 1655-1656, 1995.

[15] M. J. Hostetler, J. E. Wingate, C. J. Zhong et al., "Alkanethiolate gold cluster molecules with core diameters from 1.5 to $5.2 \mathrm{~nm}$ : core and monolayer properties as a function of core size," Langmuir, vol. 14, no. 1, pp. 17-30, 1998.

[16] S. J. Green, J. J. Stokes, M. J. Hostetler, J. Pietron, and R. W. Murray, "Three-dimensional monolayers: nanometer-sized electrodes of alkanethiolate-stabilized gold cluster molecules," Journal of Physical Chemistry B, vol. 101, no. 14, pp. 2663-2668, 1997.

[17] J. Fink, C. J. Kiely, D. Bethell, and D. J. Schiffrin, "Self-organization of nanosized gold particles," Chemistry of Materials, vol. 10, no. 3, pp. 922-926, 1998.

[18] R. H. Terrill, T. A. Postlethwaite, C. Chen et al., "Monolayers in 3 dimensions-electron-transport dynamics in solid alkanethiolstabilized gold colloids," Abstracts of Papers of the American Chemical Society, vol. 210, 380-INOR, 1995.

[19] A. Badia, L. Cuccia, L. Demers, F. Morin, and R. B. Lennox, "Structure and dynamics in alkanethiolate monolayers selfassembled on gold nanoparticles: a DSC, FT-IR, and deuterium NMR study," Journal of the American Chemical Society, vol. 119, no. 11, pp. 2682-2692, 1997.

[20] M. J. Hostetler and R. W. Murray, "Colloids and self-assembled monolayers," Current Opinion in Colloid \& Interface Science, vol. 2, no. 1, pp. 42-50, 1997.

[21] M. J. Hostetler, J. J. Stokes, and R. W. Murray, "Infrared spectroscopy of three-dimensional self-assembled monolayers: nalkanethiolate monolayers on gold cluster compounds," Langmuir, vol. 12, no. 15, pp. 3604-3612, 1996. 

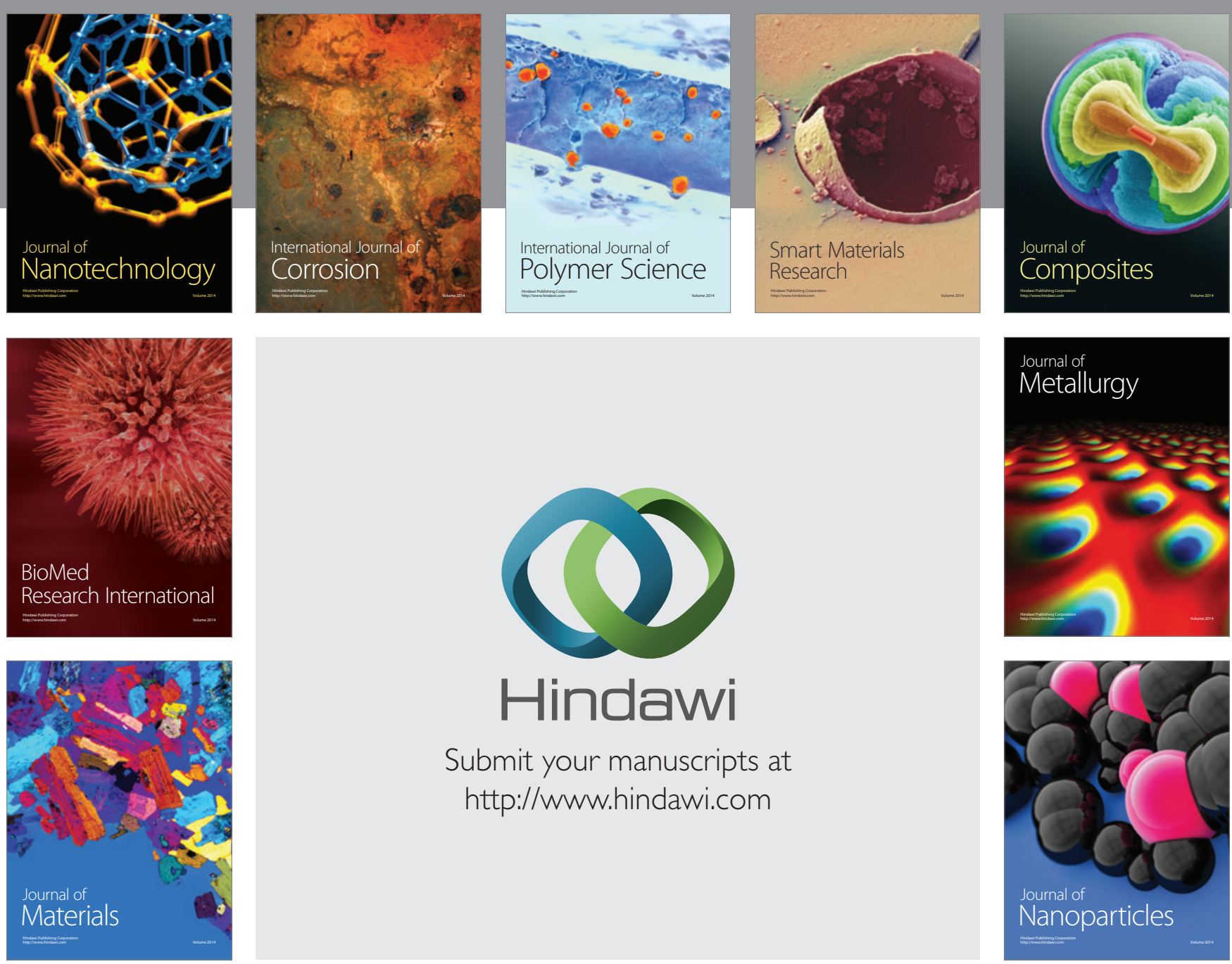

Submit your manuscripts at http://www.hindawi.com
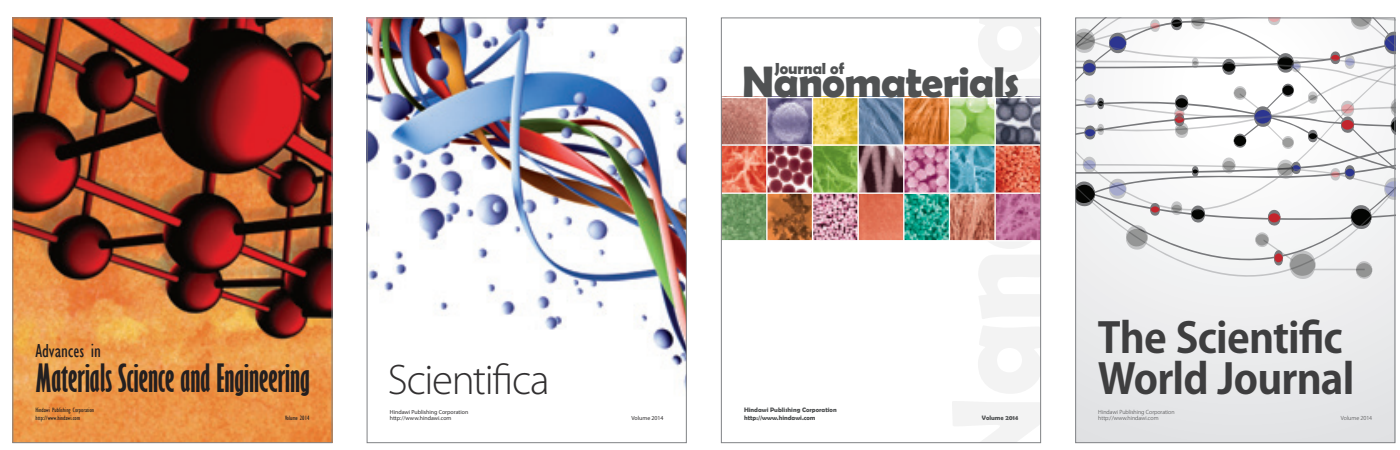

\section{The Scientific World Journal}
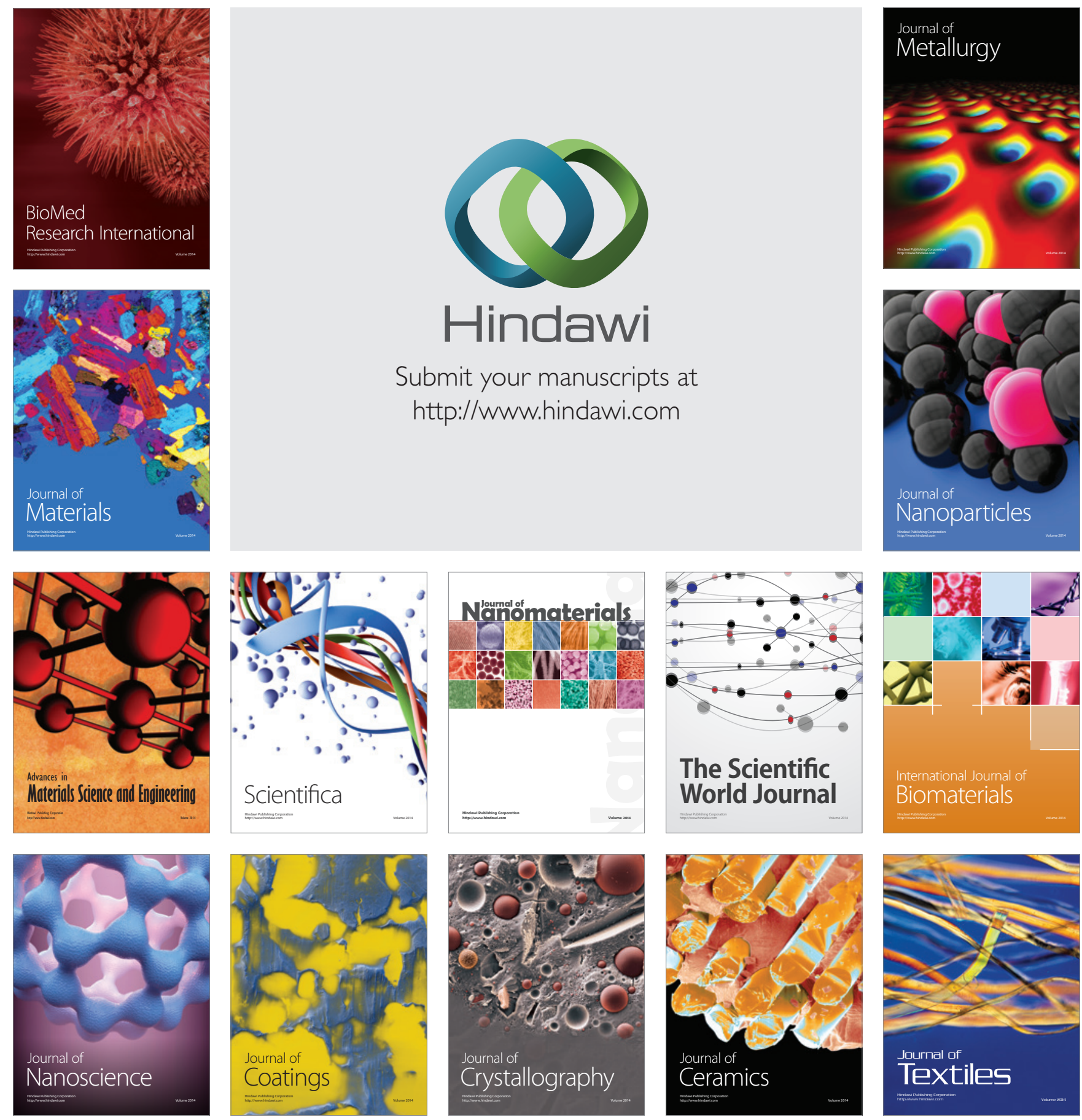